\title{
Trend of emergency department presentations for influenza like illness: Differences between pandemic (2009-2010) and post-pandemic (2010-2011) season
}

\author{
Elena Di Tondo ${ }^{1}$, Anna Marigliano ${ }^{1}$, Ilaria Pellegrini ${ }^{1}$, Francesco Di Stanislao $^{1}$, \\ Marcello M. D'Errico ${ }^{1,2^{*}}$

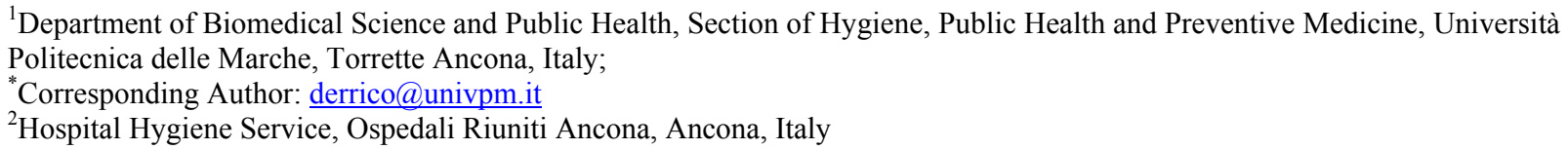

Received 22 October 2012; revised 23 November 2012; accepted 29 November 2012

\begin{abstract}
The aim of this paper is to analyze differences between incidence, characteristics and outcomes of patients with Influenza Like IIIness (ILI) presenting to a teaching public hospital in Central Italy during pandemic influenza season (20092010) and during the post-pandemic outbreak (2010-2011). We performed a retrospective descriptive study, and we identified Emergency Department (ED) presentations for ILI, relying on hospital discharge data administrative database. Bivariate analyses for the outcome of hospital admission were performed for both seasons and a multiple logistic regression model has been developed to evaluate which factors were independently associated with hospital admission. Among all the ED presentations $(51,757)$, visits for ILI were respectively 821 (3.1\%) during the pandemic season, and $704(2.8 \%)$ during the postpandemic one. The pandemic cohort differs significantly from the post-pandemic cohort for every characteristic investigated, with the exception of the length of stay in the ED. Overall in 2009-2010 there were more ED presentations compared to the following season, but hospital admission rates were less than in 2010-2011. Further studies are needed to assess if other variables may affect the use of the ED for ILI in order to try to plan staff and hospital organization.
\end{abstract}

Keywords: Influenza Like Illness; Pandemic Season; Emergency Department

\section{INTRODUCTION}

Seasonal influenza outbreaks are an important public health issue: in fact worldwide seasonal flu results in 3 5 million severe cases and 250,000 - 500,000 influenza related deaths per year [1]. Moreover during the Spring of 2009 a new virus (influenza A, H1N1) spread globally resulting in a pandemic flu outbreak [2].

Despite the lower diffusion, some authors have shown that the severity of influenza in post-pandemic period was greater than that observed during the pandemic season and that patients with comorbidity, especially obesity, were most frequently affected by the disease [3].

Seasonal epidemics, such as influenza outbreaks, can cause Emergency Department (ED) overcrowding, in fact every year during seasonal flu healthcare services utilization increases [4]. This is partly due to the wide distribution of even mild diseases throughout communities, that can overload outpatient healthcare services, leading to ED overcrowding [5]. During the pandemic period, significant surges in ED volumes were registered. As a matter of fact, when the news media inform the public about the potential for epidemic disease and possible complications of that disease including widespread morbidity and mortality, lager volumes of people could clog the EDs [6].

In Italy there is an active surveillance system for Influenza Like Illness (ILI) called InfluNet, where a sample of general practitioners and pediatricians report all cases of ILI among their patients during the epidemic period, in order to monitor the spread of the infection on the Italian territory [7]. In addition, Italian Ministry of Health has started a national weekly monitoring of ED presentations for ILI in order to forecast the number of ED visits [8].

The purpose of this study is to describe the characteristics and outcomes of ED presentation for flu like illnesses both during pandemic season (2009-2010) and during the post-pandemic one (2010-2011), in a public teaching hospital in Central Italy. This is a 900-bed third 
level teaching hospital and a point of reference for the entire Marches region (1,500,000 inhabitants). Every year, about 60,000 ED visits are performed.

\section{METHODS}

This is a retrospective descriptive study carried out in the ED of the teaching hospital "Ospedali Riuniti" in Central Italy. Data were collected from the hospital discharge data administrative database.

The influenza seasons were defined between $43^{\text {rd }}$ week of 2009 and $17^{\text {th }}$ week of 2010 for the first year and between $42^{\text {nd }}$ week of 2010 and $17^{\text {th }}$ week of 2011 for the second year, according to the national surveillance system InfluNet.

In order to identify ED presentations for ILI, the following ICD9-CM codes were evaluated: acute respiretory infections (460 - 466); other diseases of the upper respiratory tract $(470$ - 478), pneumonia and influenza (480 - 488); acute exacerbation of chronic obstructive pulmonary disease (491.21).

Data were grouped by gender (male, female), age group ( $\leq 14$ years, 14 - 65 years, $\geq 65$ years), triage code at the arrival and after medical visit and outcome (discharged, admitted to the hospital, left the hospital without being seen by a doctor, died in ED).

In Italy, a triage code is assigned to all patients presenting to ED. Triage assessment is based on a colorcode scale, which includes four categories: red (urgent, non-deferrable patients), yellow (critical patients, not in immediate life-threatening), green (deferrable patients) and white (non-urgent visits).

Length of stay in ED (LOS) has been defined as the interval between time of ED arrival and time of ED departure (either admission or discharge).

\section{DATA ANALYSIS}

Data were collected using Microsoft Access; Stata software package 9.0 was used for statistical analysis. Differences between groups (flu-like illnesses or non-flu like illnesses) and between years were studied with chisquare test for categorical variables; $\mathrm{t}$ Test for normally distributed data and Wilcoxon rank sum Test for nonnormally distributed data were used for continuous variables. Level of significance was set up at $\mathrm{p}<0.05$.

Bivariate analyses for the outcome of hospital admission were performed for both seasons.

Multiple logistic regression models were developed to adjust for confounding, and to evaluate which factors were independently associated with hospital admission (1 when hospital admission was present; 0 when absent).

All data used in this analysis are released in an anonymous form, therefore the approval of the competent Ethics Committee was not required for this specific study.
The significance level for variables to enter the multiple logistic regression models was set at $\leq 0.2$, and for removing them from the model at $\geq 0.4$.

\section{RESULTS}

Overall there were 51,757 ED visits during the two influenza seasons in the "Ospedali Riuniti" Hospital of Ancona: 26,259 in the 2009-2010 season, and 25,498 in the 2010-2011 season. Among these, visits for ILI were respectively 821 (3.1\%) during the pandemic season, and $704(2.8 \%)$ during the post-pandemic season. Differences in ILI presentations and non-ILI visits are shown in Table 1. In both seasons flu visits differed significantly from the other visits for severity at admission, and after visit. Moreover the outcome of the admission was different: in particular, there were less non urgent visits among flu-like presentations in both the seasons. Further, we found also a greater number of hospital admissions among visits for flu like illnesses compared to the others.

The pandemic cohort differs significantly $(p<0.05)$ from the post-pandemic cohort for every characteristic with the exception of the length of stay in the ED (Table 2). In 2009-2010 we found more ED presentations compared to the following season, and more men than women $(58.8 \%$ vs. $41.2 \%, \mathrm{p}<0.05)$ went to ED for a visit. In general patients attending to ED for flu like illness in 2009-2010 were younger with a mean age of 48 years for the pandemic cohort and 52 years for the post pandemic. In fact considering age groups, people aged 65 and more were fewer in 2009-2010 compared to the $2010-2011$ (28.1\% in $2009-2010$ vs. $34.2 \%$ in $2010-2011$ ). Concerning to the triage code of severity of the visit, data show that, during the pandemic season, presentations to the ED were milder than in the post-pandemic one. In fact, in 2009-2010, non-urgent and deferrable visits accounted for $79.2 \%$ of the total compared to $76.6 \%$ in the post-pandemic period; the proportions remained similar after medical visit, too.

In 2009-2010 hospital admission rates were lower than in $2010-2011(32.0 \%$ vs. $40.5 \%, \mathrm{p}<0.05)$ and they showed a different distribution in the reporting period, with an anticipation in 2009-2010, with respect to 2010-2011 (Figure 1). Age stratification revealed that most represented age group is over 65 (57.0\% during pandemic outbreak and $56.2 \%$ during post-pandemic outbreak) and that the mean age is 63 years in both seasons.

After adjustment for number of weekly ED presentations for ILI and severity code, multiple logistic regression analysis revealed that factors significantly associated with hospital admission in pandemic season were male gender $(\mathrm{OR}=1.65 ; 95 \% \mathrm{CI}: 1.12-2.43 \mathrm{p}<0.05)$ and age over 65 years $(\mathrm{OR}=6.41 ; 95 \% \mathrm{CI}: 4.25-9.66 \mathrm{p}$ $<0.05)$. 
Table 1. Characteristics of ED visits during 2009-2010 and 2010-2011 influenza seasons.

\begin{tabular}{|c|c|c|c|c|c|c|c|c|c|c|}
\hline \multirow{3}{*}{ ED presentation } & \multicolumn{5}{|c|}{ Season 2009-2010 } & \multicolumn{5}{|c|}{ Season 2010-2011 } \\
\hline & \multicolumn{2}{|c|}{ ILI } & \multicolumn{2}{|c|}{ No ILI } & \multirow[t]{2}{*}{${ }^{\text {a }}$-Value } & \multicolumn{2}{|c|}{ ILI } & \multicolumn{2}{|c|}{ No ILI } & \multirow[t]{2}{*}{${ }^{\mathrm{a}} \mathbf{p}$-Value } \\
\hline & $\mathbf{N}$ & $\%$ & $\mathbf{N}$ & $\%$ & & $\mathbf{N}$ & $\%$ & $\mathbf{N}$ & $\%$ & \\
\hline $\mathrm{N}, \%$ & 821 & 3.1 & 25,438 & 96.9 & & 704 & 2.8 & 24,794 & 97.2 & \\
\hline \multicolumn{11}{|c|}{ Gender } \\
\hline Male & 483 & 58.8 & 13,890 & 52.7 & * & 369 & 52.4 & 13,350 & 53.8 & \\
\hline Female & 338 & 41.2 & 11,548 & 43.8 & & 335 & 47.6 & 11,444 & 46.2 & \\
\hline \multicolumn{11}{|c|}{ Age (years) } \\
\hline Mean & \multicolumn{2}{|c|}{48} & \multicolumn{2}{|c|}{50} & * & \multicolumn{2}{|c|}{52} & \multicolumn{2}{|c|}{50} & * \\
\hline Standard Deviation & \multicolumn{2}{|c|}{20.8} & \multicolumn{2}{|c|}{21.0} & & \multicolumn{2}{|c|}{20.7} & \multicolumn{2}{|c|}{21.0} & \\
\hline \multicolumn{11}{|c|}{ Age group (years) } \\
\hline$\leq 14$ years & 7 & 0.9 & 146 & 0.6 & & 1 & 0.1 & 136 & 0.5 & * \\
\hline $14-65$ years & 583 & 71.0 & 18,056 & 71.0 & & 462 & 65.6 & 17,499 & 70.6 & \\
\hline$\geq 65$ years & 231 & 28.1 & 7236 & 28.4 & & 241 & 34.2 & 7159 & 28.9 & \\
\hline \multicolumn{11}{|c|}{ Triage color code at admission } \\
\hline White & 86 & 10.5 & 3446 & 13.1 & & 55 & 7.8 & 2,629 & 10.6 & \\
\hline Green & 564 & 68.7 & 17,431 & 66.1 & * & 480 & 68.2 & 17,315 & 69.8 & * \\
\hline Yellow & 161 & 19.6 & 4155 & 15.8 & & 166 & 23.6 & 4552 & 18.4 & \\
\hline Red & 10 & 1.2 & 406 & 1.5 & & 3 & 0.4 & 298 & 1.2 & \\
\hline \multicolumn{11}{|c|}{ Triage color code after visit } \\
\hline White & 62 & 7.6 & 2780 & 10.5 & & 30 & 4.3 & 2411 & 9.7 & \\
\hline Green & 570 & 69.4 & 18,882 & 71.6 & * & 475 & 67.5 & 18,861 & 76.1 & * \\
\hline Yellow & 175 & 21.3 & 25,438 & 96.5 & & 195 & 27.7 & 3196 & 12.9 & \\
\hline Red & 14 & 1.7 & 400 & 1.5 & & 4 & 0.6 & 326 & 1.3 & \\
\hline \multicolumn{11}{|c|}{ Outcome } \\
\hline Discharged & 557 & 67.8 & 20,779 & 78.8 & & 419 & 59.5 & 19,976 & 80.6 & \\
\hline Admitted & 263 & 32.0 & 3860 & 14.6 & $*$ & 285 & 40.5 & 3929 & 15.8 & * \\
\hline Left without being seen & 1 & 0.1 & 779 & 3.0 & & 0 & 0.0 & 866 & 3.5 & \\
\hline Died in ED & 0 & 0.0 & 20 & 0.1 & & 0 & 0.0 & 23 & 0.1 & \\
\hline \multicolumn{11}{|c|}{ Length of stay (min) } \\
\hline Mean & \multicolumn{2}{|c|}{220.14} & \multicolumn{2}{|c|}{215.02} & & \multicolumn{2}{|c|}{229.37} & & & \\
\hline Standard Deviation & & & & & & & & & & \\
\hline Median & & & & & * & & & & & * \\
\hline Interquartile range & 110.00 & 274.00 & 93.00 & 261.00 & & 110.00 & 80.00 & 98.00 & 67.00 & \\
\hline
\end{tabular}

${ }^{\mathrm{a}} \mathrm{p}$-Value for difference between proportions: ${ }^{*} \mathrm{p}<0.05$. 
Table 2. Comparison between flu-like illness presentations in 2009-2010 and 2010-2011.

\begin{tabular}{|c|c|c|c|c|c|}
\hline \multirow{3}{*}{ ED presentations } & \multicolumn{4}{|c|}{ Flu like illnesses } & \multirow{3}{*}{${ }^{\text {a }}$ p-Value } \\
\hline & \multicolumn{2}{|c|}{ 2009-2010 } & \multicolumn{2}{|c|}{ 2010-2011 } & \\
\hline & $\mathbf{N}$ & $\%$ & $\mathbf{N}$ & $\%$ & \\
\hline $\mathrm{N}, \%$ & 821 & 3.1 & 704 & 2.8 & * \\
\hline \multicolumn{6}{|c|}{ Gender } \\
\hline Male & 483 & 58.8 & 369 & 52.4 & * \\
\hline Female & 338 & 41.2 & 335 & 47.6 & \\
\hline \multicolumn{6}{|c|}{ Age (years) } \\
\hline Mean & \multicolumn{2}{|c|}{48} & \multicolumn{2}{|c|}{52} & * \\
\hline Standard Deviation & \multicolumn{2}{|c|}{20.8} & \multicolumn{2}{|c|}{20.7} & \\
\hline \multicolumn{6}{|c|}{ Age group (years) } \\
\hline$\leq 14$ years & 7 & 0.9 & 1 & 0.1 & \\
\hline $14-65$ years & 583 & 71.0 & 462 & 65.6 & ${ }^{*}$ \\
\hline$\geq 65$ years & 231 & 28.1 & 241 & 34.2 & \\
\hline \multicolumn{6}{|c|}{ Triage color code at admission } \\
\hline White & 86 & 10.5 & 55 & 7.8 & \\
\hline Green & 564 & 68.7 & 480 & 68.2 & * \\
\hline Yellow & 161 & 19.6 & 166 & 23.6 & \\
\hline Red & 10 & 1.2 & 3 & 0.4 & \\
\hline \multicolumn{6}{|c|}{ Triage color code after visit } \\
\hline White & 62 & 7.6 & 30 & 4.3 & \\
\hline Green & 570 & 69.4 & 475 & 67.5 & * \\
\hline Yellow & 175 & 21.3 & 195 & 27.7 & \\
\hline Red & 14 & 1.7 & 4 & 0.6 & \\
\hline \multicolumn{6}{|c|}{ Outcome } \\
\hline Discharged & 557 & 67.8 & 419 & 59.5 & \\
\hline Admitted & 263 & 32.0 & 285 & 40.5 & * \\
\hline Left without being seen & 1 & 0.1 & 0 & 0.0 & \\
\hline Died in ED & 0 & 0.0 & 0 & 0.0 & \\
\hline \multicolumn{6}{|c|}{ Length of stay (min) } \\
\hline Mean & \multicolumn{2}{|c|}{220.14} & \multicolumn{2}{|c|}{229.37} & \\
\hline Standard Deviation & \multicolumn{2}{|c|}{188.33} & \multicolumn{2}{|c|}{200.22} & \\
\hline Median & \multicolumn{2}{|c|}{176.30} & \multicolumn{2}{|c|}{188.00} & \\
\hline Interquartile range & \multicolumn{2}{|c|}{$110.00-274.00$} & \multicolumn{2}{|c|}{$110.00-280.00$} & \\
\hline
\end{tabular}

${ }^{\mathrm{a}} \mathrm{p}$-Value for difference between proportions: ${ }^{*} \mathrm{p}<0.05$. 


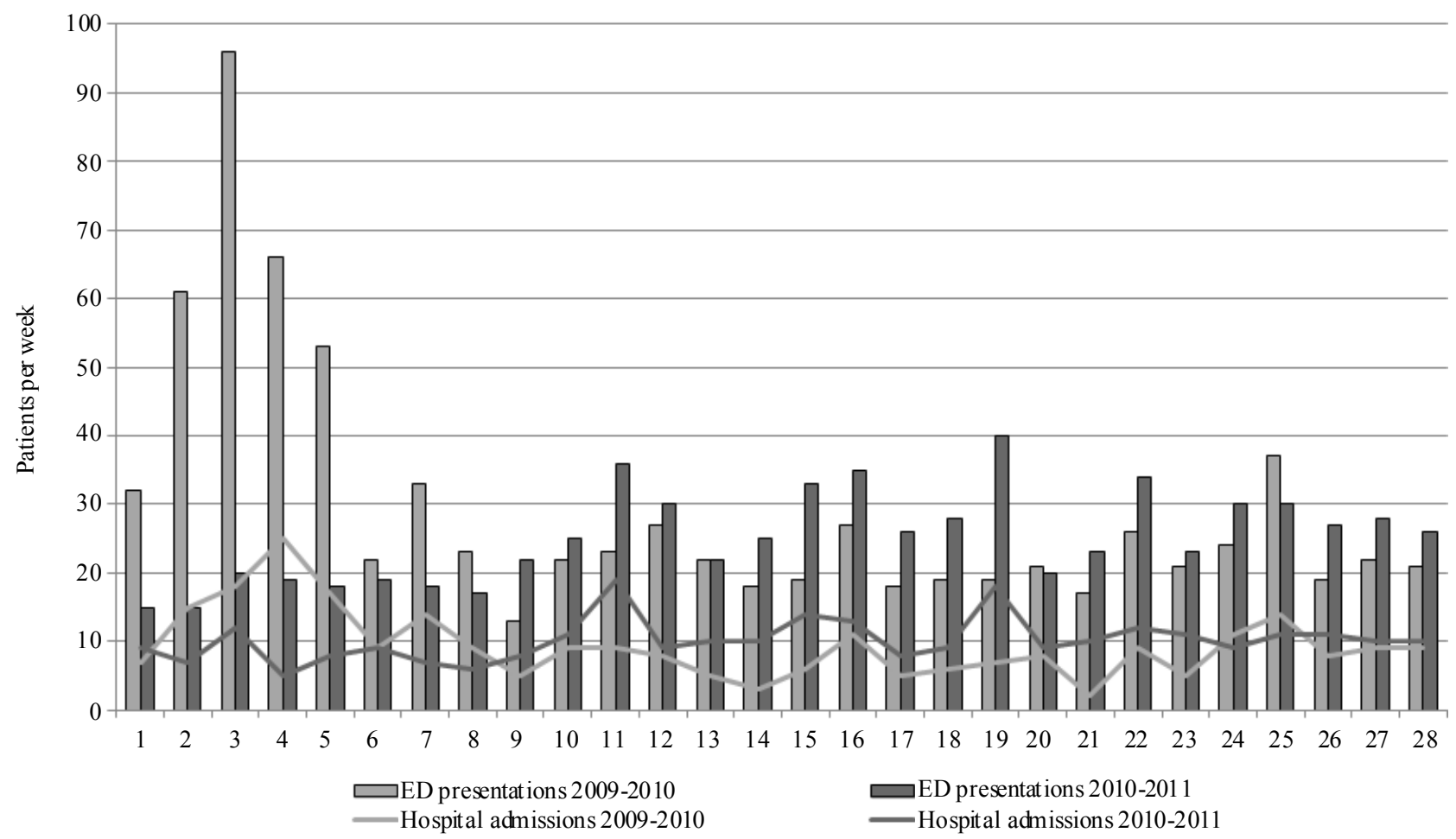

Figure 1. Distribution of number of ED presentations and hospital admissions for every week during the 2009-2010 flu season.

Regarding to the post-pandemic season, only age over 65 years was statistically significant $(\mathrm{OR}=3.85 ; 95 \% \mathrm{IC}$ : $2.67-5.56 \mathrm{p}<0.05)$.

\section{DISCUSSION AND CONCLUSIONS}

The analysis of ED visits for ILI during pandemic and post pandemic flu season has shown that they accounted for $3.1 \%$ of ED presentations during the 2009-2010 season and $2.8 \%$ during the 2010-2011 period, respectively. In line with the existing literature [9], our findings show that the percentage of outpatient visits for ILI were lower during the 2010-2011 season than the pandemic one. However, as shown in other studies [10] compared to the pandemic period, during the post pandemic season, we found a greater number of hospitalizations.

In both seasons we found that the mean age of patients attending to the ED was fairly low (mean 50 years) and that men have made a greater use of ED than women.

In Ancona city there are two specialized hospital dedicated to maternal and child health and to the management of geriatric diseases provided respectively with ED and first level ED.

This can explain both the general prevalence of use of ED by men and low representation of extreme age groups (under 14, and over 65).

Besides, patients referring to the ED during the pandemic season were significantly younger than the others attending during the seasonal influenza outbreak; in fact, as known in literature, most cases of pandemic flu infec- tion occurred in children and young adults [2,11], while the relative sparing of adults older than 60 years could probably be associated to a previous exposure to an antigenically related flu virus [12].

Boyle et al. in their study carried out in 27 ED in Australia have compared ED presentations for ILI to all the other visits in a 5-year period and have found that influenza patients were less urgent than the non-influenza cohort. Only during the pandemic season criticality/triage levels of admitted cohort was non different proportionally to the other admissions [13]. In our sample, comparing ILI visits with all the others, we found a significant lower proportion of red code at triage, but a higher yellow (critical patients) code at the admission especially during post-pandemic season. This is partially due to the fact that the Ancona Hospital is a referral center for more severe specialist diseases and for major trauma.

The analysis of the visits for a condition with a high impact in the general population such as influenza is particularly useful in health services organization, and the above data are the witness of this burden.

Vaccination is still the main strategy to prevent seasonal influenza spread. In Italy immunization against influenza is strongly recommended for people aged 65 and over and for certain groups at risks and vaccine is delivered free of charge to subjects belonging to recommended categories in order to reduce social inequities in flu immunization [14]. 
However, data from the Italian survey "Health and use of health care in Italy", carried out from December 2004 and September 2005, have shown that only $62.6 \%$ of respondents aged 65 and older reported being vaccinated against influenza [15], far from the minimum target set by Italian Ministry of Health (75\%) among older people [16]. The logistic analysis demonstrated that older age, presence of chronic disease, lower social class and more general practitioners home visits are associated with a higher likelihood of being vaccinated. In particular, being aged 85 and over $(\mathrm{OR}=1.81 ; 95 \% \mathrm{CI}$ : $1.82-2.14)$ and suffering from a severe chronic disease $(\mathrm{OR}=1.97$; 95\% CI: $1.82-2.14$ ) were the strongest predictors of vaccine uptake [15].

Furthermore in Italy vaccine coverage among adults suffering chronic obstructive pulmonary disease (COPD) is very low: less than one adult suffering from COPD out of three receives influenza vaccine. The rate of vaccinetion is even lower among adults with COPD but no other comorbidities [17], so we can assume a high probability of COPD exacerbation.

In addition influenza virus, along with other viruses such as measles, mumps and rubella, rarely may result in major complications such as encephalitis [18]. In a previous paper, we analyzed trends of hospitalization for encephalitis in Italy and we found that hospitalization associated with "viral encephalitis not otherwise specified" is increased over the period 1999-2005 [19]. So, we might suppose that this category of encephalitis could include also those developed after a complicated influenza virus infection.

Further studies are needed to verify if other differences during years in the use of ED for influenza-like illness may arise and whether other variables may affect the different use of the structure for different conditions and during the flu season in order to try to forecast patients units, health care workers staffing and beds availability.

\section{REFERENCES}

[1] World Health Organization (2009) Fact sheet No 211, influenza (seasonal). http://www.who.int/mediacentre/factsheets/fs211/en

[2] Novel Swine-Origin Influenza A (H1N1) Virus Investigation Team (2009) Emergence of a novel swine-origin influenza A (H1N1) virus in humans. The New England Journal of Medicine, 360, 2605-2615. doi:10.1056/NEJMoa0903810

[3] Rodriguez, A., Martin-Loeches, I., Bonastre, J., Olachea, P., Alvarez-Lerma, F., Zaragozae, R., Guerrerof, J., Blancog, J., Gordoh, F., Pozoi, F., Lorentej, J., Carratalák, J., Corderol, M., Rellom, J., Estebani, A., Leónn, C. and Semicyuc-Ciberes-Reipi Working Group. (2011) First influenza season after the 2009 pandemic influenza: Report of the first 300 ICU admissions in Spain. Medicina Intensiva, 35, 208-216. doi:10.1016/j.medin.2011.03.001

[4] Neuzil, K.M., Maynard, C., Griffin, M.R. and Heagerty, P. (2003) Winter respiratory viruses and health care use: A population-based study in the northwest United States. Clinical Infectious Diseases, 37, 201-207. doi:10.1086/375604

[5] Schull, M.J., Mamdani, M.M. and Fang, J. (2004) Community influenza outbreaks and emergency department ambulance diversion. Annals of Emergency Medicine, 44, 61-67. doi:10.1016/j.annemergmed.2003.12.008

[6] McDonnell, W.M., Nelson, D.S. and Schunk, J.E. (2012) Should we fear "flu fear" itself? Effects of H1N1 influenza fear on ED use. American Journal of Emergency Medicine, 30, 275-282. doi:10.1016/j.ajem.2010.11.027

[7] Italian National Institute of Health. InfluNet: Epidemiological surveillance. http://www.iss.it/flue

[8] Italian Ministry of Health. Surveillance system for influenza like illness emergency departments presentations. http://www.salute.gov.it/influenza/paginaInternaMenuInfl uenza.jsp?id=2370\&menu $=$ strumentieservizi

[9] Morbidity and Mortality Weekly Report (2011) Update: Influenza activity, United States, 2010-2011 season, and comparison of the 2011-2012 influenza vaccine, 60, 705-712.

[10] Chuang, J.H., Huang, A.S., Huang, W.T., Liu, M.T., Chou, J.H., Chang, F.Y. and Chiu, W.T. (2012) Nationwide surveillance of influenza during the pandemic (2009-10) and post-pandemic (2010-11) periods in Taiwan. PLOS ONE, 7, Article ID: e36120. doi:10.1371/journal.pone.0036120

[11] Sullivan, S.J., Jacobson, R.M., Dowdle, W.D. and Poland, G. (2010) 2009 H1N1 influenza. Mayo Clinic Proceedings, 85, 64-76. doi:10.4065/mcp.2009.0588

[12] Writing Committee of the WHO Consultation on Clinical Aspects of Pandemic (H1N1) 2009 Influenza (2010) Clinical aspects of pandemic 2009 Influenza A (H1N1) virus infection. The New England Journal of Medicine, 362, 1708-1719.

[13] Boyle, J., Crilly, J., Keijzers, G., Wallis, M., Lind, J., Sparks, R. and Ryan, L. (2012) Impact of influenza across 27 public emergency departments in Australia: A 5-year descriptive study. Emergency Medicine Journal, 29, 725731. doi:10.1136/emermed-2011-200230

[14] Chiatti, C., Barbadoro, P.,Lamura, G., Di Stanislao, F. And Prospero, E. (2011) Improving the delivery of flu vaccine for the older people in times of economic recession. Human Vaccines, 7, 986-988. doi: $10.4161 /$ hv.7.9.16693

[15] Chiatti, C., Di Rosa, M., Barbadoro, P., Lamura, G., Di Stanislao, F. and Prospero, E. (2010) Socioeconomic determinants of influenza vaccination among older adults in Italy. Preventive Medicine, 51, 332-333. doi:10.1016/i.ypmed.2010.06.008

[16] Italian Ministry of Health. Vaccination coverage. http://www.salute.gov.it/influenza/paginaInternaMenuInfl uenza.jsp?id $=679 \&$ menu $=$ strumentieservizi

[17] Chiatti, C., Barbadoro, P., Marigliano, A., Ricciardi, A., Di Stanislao, F. and Prospero, E. (2011) Determinants of 
influenza vaccination among adult and older Italian population with chronic obstructive pulmonary disease. Human Vaccines, 7, 1021-1025. doi:10.4161/hv.7.10.16849

[18] Stahl, J.P., Mailles, A., Dacheux, L. and Morand, P. (2011) Epidemiology of viral encephalitis in. Médecine et Maladies Infectieuses, 41, 453-464. doi:10.1016/j.medmal.2011.05.015

[19] Barbadoro, P., Marigliano, A., Ricciardi, A., D’Errico, M.M. and Prospero, E. (2012) Trend of hospital utilizetion for encephalitis. Epidemiology \& Infection, 140, 753764. doi:10.1017/S0950268811001002 